\title{
LIVER SEGMENTATION FOR HEPATIC LESIONS DETECTION AND CHARACTERISATION
}

\author{
C.Platero , J.M. Poncela, P. González , M.C. Tobar , J. Sanguino , G. Asensio , E. Santos \\ Applied Bioengineering Group (Universidad Politécnica de Madrid) \\ Radio-diagnostic Service, San Carlos Clinic Hospital
}

\begin{abstract}
The detection and characterisation of hepatic lesions is fundamental in clinical practice, from the diagnosis stages to the evolution of the therapeutic response. Hepatic magnetic resonance is a usual practice in the localization and quantification of lesions. Automatic segmentation of the liver is illustrated in $T_{1}$ weighted images. This task is necessary for detecting the lesions. The proposed liver segmentation is based on 3D anisotropic diffusion processing without any control parameter. Combinations of edge detection techniques, histogram analysis, morphological post-processing and evolution of an active contour have been applied to the liver segmentation. The active contour evolution is based on the minimization of variances in luminance between the liver and its closest neighbourhood.
\end{abstract}

Index Terms- Liver segmentation, anisotropic diffusion, active contours

\section{INTRODUCTION}

The discovery of a lesion occupying hepatic space is a frequent clinical situation and it is necessary to establish its nature. Optimum diagnosis requires adequate knowledge of the etiological factors. It includes two essential aspects, namely, detection and characterisation. Moreover, if it is a malignant neoplasm, other objectives are the degree of malignancy, the temporal evolution of the tumour, an adequate therapy and finally the evaluation of the treatment response and the monitoring of the treated patients [1].

At present, image techniques (ultrasound, $\mathrm{CT}$ and MRI), supported on occasions by contrast agents, constitute the main diagnostic pillar. In Spain, as in the rest of Europe and Japan, ultrasound imaging is used as initial diagnosis, while computerized tomography and magnetic resonance are used as methods of characterisation and evolution of the lesions.

Hepatic MRI is a new diagnostic method which has experienced important advances. It produces high quality images. Such advances include rapid scanning, new sequences of volume with a very high spatial resolution and more specific contrasts for each type of lesion [1].

MRI seems to be the appropriate diagnostic procedure for benign tumours, such as haemangioma and focal nodular hyperplasia. In hepatocellular carcinomas, MRI with gadolinium probably achieves better results than $\mathrm{CT}$, although with limitations in the characterisation of the lesions (50\% specificity) and it appears to be slightly insensitive for detecting satellite nodules $(50-70 \%$ sensitivity). MRI with specific contrast behaves better than $\mathrm{CT}$ in patients with hepatic metastasis of reduced size [2].

\section{MOTIVATION}

Current commercial systems offer tools for manual segmentation of lesions. Usually, the radiologist selects the areas of interest with the mouse and semi-automatic techniques adjust the contour for each slice. However, automatic segmentation techniques are encouraged and justified in terms of the volume of information handled, the required amount of expert time and the repetitive and unrewarding nature of the task. Recently, it has been demonstrated that a bias exists between the clinical practice of inferred measurements of hepatic lesions based on onedimensional or two-dimensional criteria and volume measurement analysis [3]. The results of semi-automatic techniques, using watershed and active contours, guarantee the robustness and reliability of these methods compared to manual segmentation ([4], [5]).

Automatic detection and characterisation of hepatic lesions requires first segmentation of the liver. This delimitates the target space. Then, lesions are distinguished from parenchyma and vascular system via appearance and shape.

Although many works have been produced since the nineties, especially in $\mathrm{CT}$, the variance in liver size and shape between the patients and the proximity with other organs of similar intensity make automatic liver segmentation especially difficult([6], [7]). We have to add to this situation the inherent problems to magnetic resonance images, such as the lack of homogeneity in the radiated magnetic field and the artefacts of the coils. These problems produce a strong variation in the grey level inside the organ. 
Ibrahim et al [8] have modelled the MRI 2D signal as well as the addition of multiplicative and Gaussian noise. To filter it, they have presented a combined application of a local enhanced filter followed by a median. In this presentation, we propose, as part of the success of automatic liver segmentation, the application of a 3D anisotropic diffusion for the processing of MRI abdominal images.

In the following section we describe the fundamentals of the processing techniques we have used. The fourth section deals with segmentation algorithms. Finally, the numerical methods employed and the experiments are introduced in section five.

\section{PROCESSING BASED ON 3D ANISOTROPIC DIFFUSION}

The application of a non-linear diffusion filter on an image in grey levels, $u_{0}: \Omega \subset \mathbb{R}^{n} \rightarrow \mathbb{R}$, generates another processed image, $u(x, t)$, as a solution of the diffusion process with Neumann and initial contour conditions:

$$
\begin{array}{ccc}
\partial_{t} u=\operatorname{div}(g(|\nabla u|) \nabla u) & x \in \Omega & t>0 \\
u(x, 0)=u_{0}(x) & x \in \Omega & \text { c.i. } \\
\partial_{x} u=0 & x \in \partial \Omega & \text { c.c. }
\end{array}
$$

As $g(|\nabla u|)$ we can choose a family of uniparametric diffusivities without any control parameter [9]:

$$
g(|\nabla u|)=\frac{1}{|\nabla u|^{p}} \quad p \in \mathbb{R}^{+}
$$

For $p>1$ it is characterised by a combination of direct diffusion in the tangential component to the level curve and inverse diffusion in the normal direction. For $p=2$, the diffusion process enhances the edges, in a wide variety of slope ranges, and smoothing of homogeneous areas.

\subsection{Numerical filter}

Although originally the filter comes from a convex functional in its continuous expression, it can be unconditionally stable in its discretization process. For the $1 \mathrm{D}$ case, the diffusive nature of the equation (1) may have an approximation of the spatial derivative of the form

$$
\begin{gathered}
\partial_{t} u=\partial_{x}\left(g\left(\left|\partial_{x} u\right|\right) \partial_{x} u\right) \approx \\
\frac{1}{2}\left[\delta_{x}^{+}\left[g\left(\delta_{x}^{-} u\right)\right] \delta_{x}^{-} u+\delta_{x}^{-}\left[g\left(\delta_{x}^{+} u\right)\right] \delta_{x}^{+} u\right],
\end{gathered}
$$

$\delta_{x}^{+}$and $\delta_{x}^{-}$being the progressive and regressive differences, obtaining the semi-discrete expression

$$
\begin{aligned}
& \frac{d u_{i}}{d t}(t)=\frac{1}{h^{2}} \\
& \quad\left[g_{i+1}(t)\left(u_{i+1}(t)-u_{i}(t)\right)-g_{i-1}(t)\left(u_{i}(t)-u_{i-1}(t)\right)\right]
\end{aligned}
$$

The matrix form would be

$$
\frac{d[u]}{d t}=A(u)[u]
$$

where

$$
a_{i j}(u)=\left\{\begin{array}{cc}
\frac{g_{i j}}{h^{2}} & j \in N(i) \\
-\sum_{j \in N(i)} \frac{g_{i j}}{h^{2}} & i=j \\
0 & j \notin N(i)
\end{array}\right.
$$

with $h=\Delta x$ and $N(i)$ being the set of the two pixels closer horizontally to $i$ (only one if $i$ is a contour pixel). As a result, this filtering process produces a matrix $A$ which satisfies the conditions for evolution within a scale-space frame [10]. Finally, the stability condition on the time step size can be lifted with a semi-implicit scheme, $\Delta t \equiv k$. For such purpose a time step is taken to be $k>0$ and is represented by $u_{i}^{m}$ an approximation of $u\left(x_{i}, m k\right)$, giving the equation,

$$
\frac{u_{i}^{m+1}-u_{i}^{m}}{k}=\sum_{j \in N(i)} \frac{g_{i j}^{m}}{h^{2}}\left(u_{j}^{m+1}-u_{i}^{m+1}\right)
$$

In matrix form,

$$
\left[I-k A\left(u^{m}\right)\right]\left[u^{m+1}\right]=\left[u^{m}\right] .
$$

Extension to a dimension greater than one is achieved through decomposition of the diffusion process into its components based on AOS (Additive Operator Splitting) [10].

\section{SEGMENTATION ALGORITHM}

Once the volume of interest has been defined, where the liver is located, we can proceed to the $3 \mathrm{D}$ diffusion process above mentioned. The resulting image shows enhanced edges and smoothed homogeneous areas. From the histogram analysis of the filtered image, we obtain a low and a high threshold which are capable of defining the liver tissue. However, the multiplicative noise which appears in the MRI signal requires thresholds which are relatively unrestricted, producing an overlapping of tissue with the hepatic vascular system and other adjacent organs. A Canny detector is used to detect close contours on the filtered image. Next, the performance of morphological filtering in the threshold image and contour detection image reduces the object of the liver, preserves the shape of the liver, and detects the initial liver region. To reduce the noise and detect the coarse liver region, we perform on the regionlabelling algorithm. Since the liver is the largest organ, we can select the first approximation of its segmentation.

After this first splitting of the image, a part of vascular system is outside the course segmentation and the liver contour belong to each slice is blur and inaccurate. Starting 
with this first solution, an active contour is evolved in order to final segmentation.

\subsection{Final segmentation}

The liver course segmentation contains mainly parenchyma. A large part of the hepatic vascular system, the external musculature and the right kidney are left outside. The starting hypothesis for refinement is based on the fact that the closest neighbourhood has lower intensity than the liver. This affirmation is true in the protocols which are used for hepatic lesions detection and that are based on 3D axial dynamic analysis. The contour will evolve inside the liver with an average growth in intensity when it adds the vascular system. Therefore, the intensity variability inside the liver will increase, and must be neutralised by external variability close to the liver.

We propose to apply a simplified Mumford-Shah functional. The objective is the minimization of internal and external variance in luminance with respect to the partition of the contour together with minimization of the contour. Let $C \subset \mathbb{R}^{n-1}$ be the boundary hypersurface of the open subset $\omega \subset \Omega$, which represents the segmented liver. The energy level of the contour is defined as [11]

$$
\begin{gathered}
E\left(c_{\text {in }}, c_{\text {out }}, C\right)=\lambda_{\text {in }} \int_{\omega}\left(u-c_{\text {in }}\right)^{2} d x+ \\
\lambda_{\text {out }} \int_{\Omega / \bar{\omega}}\left(u-c_{\text {out }}\right)^{2} d x+\mu H^{n-1}(C)
\end{gathered}
$$

where $c_{\text {in }}$ and $c_{\text {out }}$ are the average intensity values inside and outside the contour:

$$
c_{\text {in }}=\frac{\int_{\bar{\omega}} u d x}{\int_{\bar{\omega}} d x} \quad c_{\text {out }}=\frac{\int_{\Omega \backslash \bar{\omega}} u d x}{\int_{\Omega \backslash \bar{\omega}} d x}
$$

Moreover $H^{n-1}(C)$ denotes the measurement of $C$ in the dimension $n-1$, and $\lambda_{\text {in }}>0, \lambda_{\text {out }}>0$ y $\mu>0$ are control parameters.

\section{RESULTS}

The FSPGR sequence was used in the $T_{1}$ weighted images of the patients and the breath-hold protocols were followed in order to eliminate movement artefacts. ITK was used to develop the algorithms and clinical practice was conducted with the necessary modifications in ITK-Snap.

In the diffusion process, the unlimited diffusivity when the gradient module approaches infinity has been resolved by using a small regularization constant $\varepsilon$. The value of $10^{-3}$ has been used in the experiments. The AOS algorithm has been used to resolve the semi-implicit scheme. The images have been filtered with a single iteration with a time increment of $0.01 \mathrm{~s} \mathrm{y} p=2$. Figure 1 shows four consecutive sections of the liver. The original image is shown on the left and the enhanced image on the right.

The use of level set techniques for active contour implementation has become very popular, due to its handling of points presenting discontinuities and the possibility of topological change. However, the numerical stability problems of the dynamic and their high computational cost are considered to place important limitations in practice. Recently, a new method, included in the narrow band techniques, allows the elimination of the re-initialization stages, reduces the bandwidth to a $3 \times 3 \times 3$ environment and uses a simple scenario of finite differences [12]. It is based on the addition of a term of preservation of the signed distance function in the level set. Moreover, the initialization of the contour may be based on a binary image, allowing the integration of classic processing and segmentation techniques as an starting element. The active contour computational cost is reduced at least in two orders of magnitude.

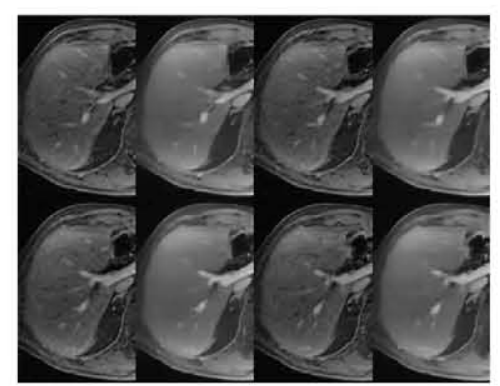

Figure 1. Four sections of the liver. On the left, the original image, on the right, the filtered image

Figure 2 shows a series of liver sections, the initial course contour appears in green; the final contour of the evolution appears in red. Figure 3 shows the $3 \mathrm{D}$ reconstruction of the final liver segmentation.

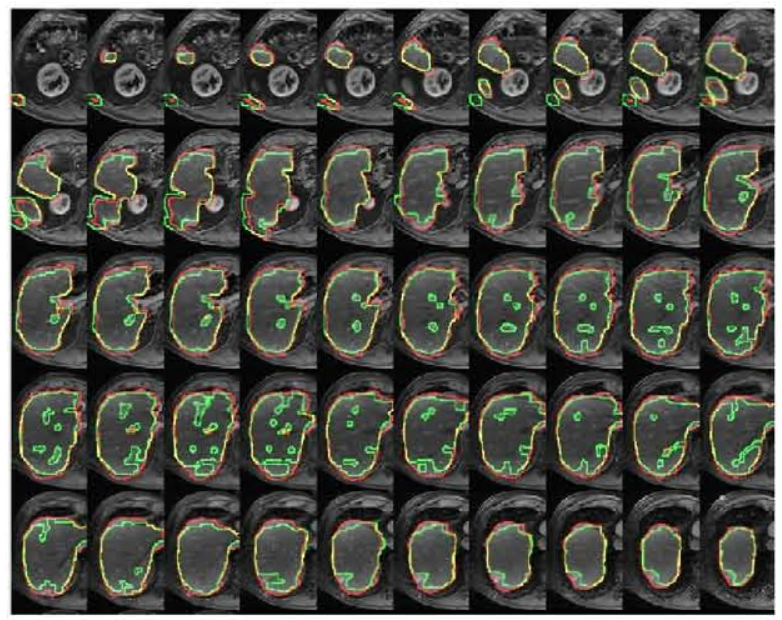

Figure 2. Solution of some liver sections. At the beginning the contour is green; the final solution is red. 
The computational cost for a volume of $350 \times 250 \times 55$ pixels is $28 \mathrm{~s}$., for diffusive filtering, thresholding and postprocessing, and $6 \mathrm{~s}$. for evolution of the active contour.

To validate the segmentation result, we compute the undirected partial Hausdorff distance [13] between the boundary of the computed segmentation and the boundary of the manual-segment ground truth. The result was $2.3 \mathrm{~mm}$ for $95 \%$ percentil and $2.8 \mathrm{~mm}$ for $99 \%$.

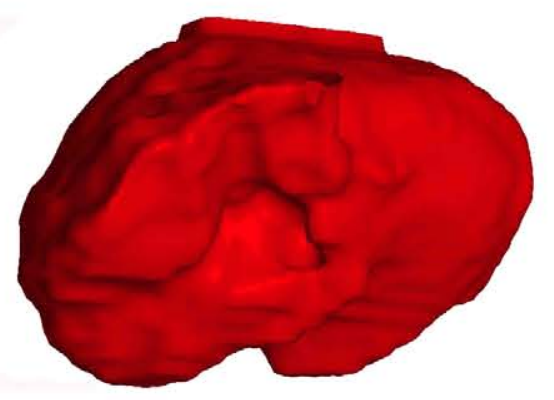

Figure 3. 3D reconstruction of the liver with 55 sections

\section{CONCLUSIONS}

We have presented a liver automatic segmentation algorithm for hepatic lesions detection. First, we apply a 3D anisotropic diffusion for image enhancement. The course segmentation is obtained by a combination of edge detection techniques, histogram analysis and binary morphological post-processing. An active contour is applied for refining course segmentation. It is based on the minimization of variances in luminance between the liver and its closest neighbourhood.

\section{ACKNOWLEDGEMENT}

This paper was supported by MEC grant DPI2007-63654.

\section{REFERENCES}

[1] C. Bartolozzi, C. Della Pina, D. Cioni, L. Crocetti, E. Batini, R. Lencioni, "Magnetic Resonance: Focal Liver Lesions Detection, Characterization, Ablation", Medical Radiology, Springer, Berlin, 2005 .

[2] R. Lencioni, D. Cioni, L. Crocetti L, "Review. Magnetic resonance imaging of liver tumors" J Hepatol, 40, pp 162-171, 2004.

[3] S.R. Prasad, K.S. Jhaveri, S. Saini, P.F. Hahn, E.F. Halpern, J.E. Sumner, "CT tumor measurement for therapeutic response assessment: comparison of unidimensional, bidimensional, and volumetric techniques initial observations", Radiology, 225(2), pp. $416-419,2000$

[4] P. Yim, D. Foran, "Volumetry of Hepatic Metastases in Computed Tomography using the Watershed and Active Contour
Algorithms", Proceedings of the 16th IEEE Symposium on Computer-Based Medical Systems (CBMS'03), 2003.

[5] R. Lu, P. Marziliano, C.H. Thng, "Liver tumor volume estimation by semi-automatic segmentation method", Proceedings of the 2005 IEEE Engineering in Medicine and Biology 27th Annual Conference, Shanghai, China, September 1-4, 2005.

[6] L. Soler, H. Delingette, G. Malandain, J. Montagnat, N. Ayache, C. Koehl, O. Dourthe, B. Malassagne, M. Smith, D. Mutter, J. Marescaux, "Fully automatic anatomical, pathological and functional segmentation from CT scans for hepatic surgery". Comput Aided Surg vol. 6, pp. 131-142, 2001.

[7] K.S. Seo, H.B. Kim, T. Park, P.K. Kim, J.A. Park, "Automatic Liver Segmentation of Contrast Enhanced CT Images Based on Histogram Processing", ICCS 2005, LNCS 3516, pp. 822 - 825, 2005 .

[8]H. Ibrahim, M. Petrou, K. Wells, S. Doran, O. Olsen, "Preprocessing for use in Automatic Volumetric Liver Segmentation from NMR Data", Nuclear Science Symposium Conference Record, IEEE, 2004.

[9] S. L. Keeling and R. Stollberger. "Nonlinear anisotropic diffusion filters for wide range edge sharpening", Inverse Problems, 18, pp 175-190, January 2002.

[10] B. Weickert, M. Haar Romeny, M.A. Viergever, "Efficient and reliable schemes for nonlinear diffusion filtering". IEEE Transactions on Image Processing, vol. 7(3), pp. 398-410, 1998.

[11] T. Chan, L. Vese, "Active contours without edges", IEEE Transactions on Image Processing, vol. 10(2), pp. 266-277, 2001.

[12] C. Li, C. Xu, C. Gui, M.D. Fox, "Level Set Evolution without Re-Initialization: A New Variational Formulation", CVPR vol. 1, pp. 430-436, 2005.

[13] D. Huttenlocher, G. Klanderman, W. Rucklidge, "Comparing images using the Hausdorff distance" IEEE Trans PAMI, 15, pp 850-863, 1993. 